\title{
Modified Overall Immune Related Response Criterion, Progressive Disease
}

National Cancer Institute

\section{Source}

National Cancer Institute. Modified Overall Immune Related Response Criterion,

Progressive Disease. NCI Thesaurus. Code C111087.

The evaluation of the antitumor response shows that the sum of the longest diameters of target and new measurable lesions increases by at least 20\% (compared to nadir), and is confirmed by repeated, consecutive observations at least 4 weeks (normally done at 6 weeks) from the date first documented. 